\title{
INFLUENCIA DE LOS SIGNOS Y SÍNTOMAS DE ESQUIZOFRENIA EN LA TEORÍA DE LA MENTE
}

\author{
Paz López-Herrero* \\ Elvira Mendoza Lara* \\ José A. Muela Martínez** \\ Sukhl S. Shergill***
}

"Departamento de Personalidad, Evaluación y Tratamientos Psicológicos. Universidad de Granada

"*Departamento de Psicología. Universidad de Jaén.

***Senior Lecturer en Institute of Psychiatry. Department of Psychiatry. Kings College London. United Kingdom

\section{RESUMEN}

La teoría de la mente propone que los signos y sintomas de la esquizofrenia reflejan una disfunción en el sistema cognitivo dedicado al reconocimiento y control de nuestras propias intenciones y a la atribución de intenciones a otras personas. Nuestra pretensión es analizar si los deficits metarepresentacionales observados en esquizofrénicos se asocian a su sintomatología. 47 sujetos se seleccionaron para el estudio: 27 esquizofrénicos y 20 sujetos control. Las pruebas utilizadas fueron: Test de factor " $g$ ", escala PANSS y tarea extensiva de verbos mentales. Los enfermos se reunieron, según sus signos y síntomas, en los grupos de: signos conductuales, sintomas

El presente trabajó se realizó en el Dpto. Personalidad, Evaluación y Tratamiento Psicológico .Universidad de Granada. España.

Correspondencia: Dra. Dña. Paz López-Herrero. Facultad de Educación y Humanidades. Campus Universitario.Dpto. Personalidad, Evaluación y Tratamiento Psicológico (Universidad de Granada. Carretera Alfonso XIII s/n. 52005 Melilla. España.Tel: 952698734/952670703. E-mail: pazlopez@ugr.es 
paranoides, fenómenos de pasividad y otros síntomas menos típicos/remisión. Se encontraron dificultades en TM en pacientes con signos conductuales negativos y positivos, con delirios paranoides y con características de paș/vidad; dificultades que se plasmarán en su discurso. Asimismo, los pacientes con signos conductuales ejecutaron la tarea peor que los pacientes con delirios paranoides. El grupo con otros síntomas menos típicos y en remisión realizaron la tarea de mentalización normalmente.

Palabras clave: TEORIA DE LA MENTE, ESQUIZOFRENIA, SINTOMATOLOGÍA, SIGNOS, SINTTOMAS, TAREA EXTENSIVA DE VERBOS MENTALES.

\section{SUMMARY}

The theory of mind proposes that the signs and symptoms of schizophrenia reflect the precise nature of the dysfunction within a cognitive system devoted to the recognition and monitoning of one 's own intentions and the attribution of intentions to others. This study examines the hypothesis that metarepresentational deficits seen in patients with schizophrenia are associated with their symptomatology. Forty- seven people were assessed during this study: twenty- seven patients with a diagnosis of schizophrenia and twenty non-psychiatric controls. Measures: Factor " $g$ " test, PANSS scale and mental verbs extension task. The patients were allocated to one of four groups according to their current signs and symptoms: behavioural signs, paranoid symptoms, passivity phenomena and other symptoms/remission. We found metarepresentational problems in patients with negative and positive behavioural signs, paranoid delusions and passivity features and associated with speech disorder. Also, our psychiatric participants with behavioural signs performed the task significantly more poorly than do patients with paranoid delusions; while the group with other symptoms and those in remission performed the task normally.

KeY WOrdS: THEORY OF MIND, SCHIZOPHRENIA, SYMPTOMATOLOGY, SIGNS, SYMPTOMS, MENTAL VERBS EXTENSION TASK. 


\section{INTRODUCCIÓN}

Existe un vínculo entre la teoría de la mente (TM) y la esquizofrenia, pues las anomalias cognitivas que subyacen a los signos y síntomas de la esquizofrenia reflejarian un defecto presente en un mecanismo fundamental para la experiencia consciente; a este mecanismo se le puede denominar metarrepresentación o conocimiento de uno mismo acerca de lo que saben los demás. Los esquizofrénicos pueden tener problemas a la hora de hacer inferencias sobre el conocimiento $\theta$ intenciones de sus oyentes, así como con el uso de tales inferencias para que les sirvan de guía en su discurso. Concretamente, Frith en 1995 propone que la esquizofrenia puede ser un trastorno de autoconsciencia en el que hubiera dificultades relacionadas con la acción voluntaria, con el autocontrol y con el control de las intenciones de los demás (Herold, Tényi, Lénárd y Trixler, 2002; Langdon y Coltheart, 2000; Langdon, Coltheart, Ward y Catts, 2002). Tales problemas se podrían clasificar como dificultades en el conocimiento de nuestras propias metas, de nuestras propias intenciones y de las intenciones de los otros (Frith, 1997).

Frith y sus colegas han utilizado un acercamiento de síntomas, dado que, frecuentemente, en la enfermedad aparecen agrupaciones de síntomas que co-ocurren conjuntamente. Estos autores distinguen entre síntomas observables de la esquizofrenia que son denominados signos conductuales y los síntomas no observables que son aquellos que los pacientes reconocen poseer y experimentar. Partiendo de la clasificación en diferentes grupos de signos y síntomas este modelo hace una serie de predicciones sobre la ejecución de dichos pacientes esquizofrénicos en tareas de TM (Corcoran 2001; Pickup y Frith, 2001):

a- Grupo con signos negativos: que se caracteriza por presentar retirada social, pobreza en el discurso, abulia y afecto embotado.

b- Grupo con signos positivos: que presenta trastorno formal del pensamiento (en cuyo lenguaje aparece descarrilamiento, neologismos, fuga de ideas, incoherencia en su discurso), conducta desorganizada y afecto incongruente.

Allen, Liddle y Frith, 1993; Corcoran, 2001; Frith, Leary, Cahill y Johnstone (1991) apoyaron la idea de que los pacientes con cualquier 
signo conductual negativo o positivo obtienen puntuaciones bajas en tareas de TM, ya que no pueden representar los estados mentales.

c- Grupo con delirios paranoides: en este grupo encontramos delirios de referencia, de persecución, de interpretación errónea de la conducta e intenciones de otras personas, de transformación de objetos y personas conocidos, de lectura de la mente y alucinaciones auditivas de persecución en tercera persona.

Corcoran (2000); Corcoran, Cahill y Frith (1997); Corcoran, Mercer y Frith (1995) y Mazza, De Risio, Roncone y Casacchia (2001) esperaban que los sujetos esquizofrénicos con sintomatología paranoide mostraran dificultades en TM, pero en menor grado que los pacientes con signos conductuales. Se podría afirmar que estos grupos de pacientes se diferencian en cuanto a la profundidad de sus problemas de TM, pues mientras que los paranoides manifiestan problemas solo en tareas más complejas de segundo orden, los pacientes con signos negativos tienen déficits más severos en TM tal y como demuestran las dificultades que aparecen en tareas de primer orden (Corcoran y Frith, 1996; Frith y Corcoran, 1996). Pero contrariamente, en los estudios de Herold, et al., (2002); Langdon et al. (1997); Mitcheley et al. (1998) no se encuentran evidencias de que los síntomas paranoides fueran asociados a una dificultad en TM y que los pacientes con tales síntomas ejecutaran peor que los controles las tareas de falsas creencias.

d- Grupo con características de pasividad: en el que se observan delirios de control y de influencia, inserción del pensamiento, robo de pensamiento y alucinaciones auditivas en segunda persona. Estos sujetos manifestaban una falta de habilidad representacional que implicaba la falta de control de sus propias intenciones con el fin de actuar; sin embargo, eran capaces de inferir las intenciones de los otros a través del discurso indirecto. Por ello, realizarán las tareas de TM normalmente (Garety y Freeman, 1999).

Corcoran (2001) obtiene unos datos diferentes, concluyendo que en los pacientes con fenómenos de pasividad se desconoce hasta qué punto un déficit como este afectaría la habilidad para reflexionar sobre los estados mentales de otras personas.

e- Grupo con otros síntomas menos típicos: entre tales síntomas destacan las alucinaciones musicales y los delirios sexuales, 
hipocondríacos y de grandiosidad. Para este grupo se puede asumir que las habilidades de TM funcionan, logrando una correcta mentalización.

f- Grupo de pacientes en remisión: compuesto por enfermos que no presentan signos o síntomas durante al menos dos semanas. En él las habilidades en TM son normales y la solución de tareas de TM es tan correcta como la que realizan los grupos control no psicóticos (Corcoran et al., 1995).En este mismo sentido, Herold et al. (2002) y Sarfati, Passerieux y Hardy-Baylé (2000) definen la falta de habilidad en TM como funcional, inconstante y relacionada con la sintomatología del trastorno de modo que algunos procesos cognitivos temporalmente funcionan de una forma anormal, lo que conlleva que el déficit en TM pueda desaparecer cuando el episodio agudo de la esquizofrenia está en remisión. El problema parece depender del estado de los pacientes, de hecho, los signos negativos, positivos y/o la sintomatología paranoide generan una ruptura temporal de las habilidades en TM (Corcoran, 2000; Drury, Robinson y Birchwood, 1998).

No obstante, también se afirma que los problemas en TM pueden ser detectados no solo durante las fases agudas sino también durante la fase de remisión de la enfermedad. Así, el modelo del desarrollo de la psicopatología predice que los sujetos esquizofrénicos realizarian bien tareas simples de TM, pero fallarían en tareas más complejas que requieren apreciación del humor, de la ironía o de la entonación para comprender los estados mentales de los demás (Herold et al., 2002). Otros autores como Mazza et al., 2001, manifiestan una idea similar y comentan que las habilidades de TM en psicosis se desarrollan normalmente, pero que tras el primer episodio de la enfermedad, se vuelven más limitadas; no obstante, también apuntan que el déficit puede mejorar cuando los síntomas psicóticos agudos remiten.

Diferentes son las tareas que facilitan el estudio de la TM, entre ellas vamos a referirnos las utilizadas por Schwanenflugel y sus colaboradores, que se ciñen al significado y uso que los niños dan a verbos relacionados con las actividades mentales. Para estos investigadores, la aparición de nuevos conceptos sobre los verbos mentales y los cambios, en la organización de dichos conceptos, que tienen lugar durante la evolución son consistentes con el desarrollo de una teoría constructivista de la mente durante los años escolares 
(Mendoza, 2001). Asimismo, destacan un empleo intencional, abstracto y relacionado con la construcción de espacios mentales separados de la realidad, que permitirian concebir las intenciones y las falsas creencias. En un sentido lingüistico, una maestría semántica de los verbos mentales se lograría a través del despliegue de las capacidades metarrepresentacionales (Bouchand y Caron, 1999).

Concretamente, la transformación de la TM en la infancia se observa en la existencia de un progreso en los aspectos de procesamiento / información de las actividades mentales, por el cual tales actividades se van organizando y extendiéndose, sin interrupción, desde lo perceptivo a lo conceptual, desde el mundo externo al mundo interno y desde la categoría de input a la categoría de output. De este modo, las personas distribuyen los verbos mentales a lo largo de un continuo denominado procesamiento de la información, que va desde las funciones de input, pasando por las funciones de procesamiento y memoria, hasta las funciones de output. Asimismo, otra característica del desarrollo de la TM es la que declara que las personas dan importancia al aspecto de certeza no sólo en función de la edad, sino también de la precisión de la comprensión en las tareas de tipo comprensivo. De hecho, las actividades de memoria son vistas como más ciertas, las de input / output se evalúan como de duda moderada, mientras que las de procesamiento son las más dudosas. Puntualizando más, son las tareas extensivas de verbos mentales, que implican un número de procesos cognitivos que operan simultáneamente por lo que en ellas es posible escoger como respuesta varias actividades mentales, las que aportan más datos sobre la comprensión de los adultos acerca de los verbos de procesamiento (Clark, Schwanenflugel, Everhart y Bartini, 1996; Moore, Pure y Furrow, 1990; Schwanenflugel, Fabricius y Alexander, 1994 a; Schwanenflugel, Fabricius y Noyes, 1996; Schwanenflugel, Henderson y Fabricius, 1998; Schwanenflugel, Martin y Takahashi, 1999). En función de estos esquemas organizativos, Schwanenflugel et al. (1994b, 1998) plantean la existencia de tres categorías en las que se agruparían los verbos mentales: Input, Memoria y Procesamiento.

El objetivo principal de esta investigación sería el estudio de la TM, utilizando, para ello, una prueba de verbos mentales en un grupo de sujetos diagnosticados con esquizofrenia (Grupo Clínico) y compa- 
rando sus respuestas con las que establece un grupo de sujetos normales (Grupo Control). Dicho objetivo se podría dividir en los siguientes sub-objetivos:

Identificar las categorías en las que se agrupan los verbos mentales, así como los verbos que las forman.

Describir el significado y el perfil de uso que se otorga a las actividades mentales.

Examinar si los signos y síntomas, que presentan los sujetos, median en la ejecución de la tarea de verbos mentales.

\section{MÉTODO}

\section{Grupos}

El Grupo Clínico lo integran 43 esquizofrénicos evaluados, diagnosticados y tratados por la Unidad de Psiquiatria del Área de Salud de Melilla. Sin embargo, y debido a que se controlaron las variables siguientes: edad máxima de 55 años, ausencia de retraso mental, auditivo, problemas neurológicos o drogadicción (sin consumo durante los últimos seis meses), formación académica mínima española (en posesión del título de Graduado Escolar o similar), asistencia a las revisiones psiquiátricas y uso de medicación, finalmente fueron 27 los pacientes seleccionados para formar parte de esta investigación. La elección de estos sujetos se realizó en función del diagnóstico psiquiátrico establecido por la mencionada Unidad, que se basó en los criterios de la Organización Mundial de la Salud para la clasificación de los trastornos mentales y del comportamiento (CIE) para los diversos tipos de esquizofrenia $(20.0, n=16.20 .3, n=2.20 .5, n=7$. 20.6, $n=2$ ). El Grupo Control está formado por 20 adultos, concretamente por estudiantes de $4^{\circ}$ de ESO del Instituto de Enseñanza Secundaria Enrique Nieto y de la Universidad de Mayores, todos en la ciudad de Melilla. Nuestra pretensión era equipar este grupo, en cuanto a edad y formación académica se refiere, con el Grupo Clínico. Los datos socio-demográficos y clínicos de los grupos aparecen en la Tabla 1. 
Tabla 1: Datos sociodemográficos y clínlcos de los particlpantes

\begin{tabular}{|llc|}
\hline & $\begin{array}{c}\text { Grpo Control } \\
(n=20)\end{array}$ & $\begin{array}{c}\text { Gupo Cínico } \\
(n=27)\end{array}$ \\
\hline Sexo V:H & $7: 13$ & 225 \\
Edad media (años) & $31^{\prime 2}(18-55)$ & $37(25-55)$ \\
Media de años de escolarización & $10^{\prime} 9$ & $11^{\prime 2}$ \\
C.l. & $105^{\prime} 8$ & $85^{\prime} 1$ \\
Duración media de la enfermedad (años) & - & $16^{\prime 2} 2(2-35)$ \\
Dosis media de medicación (Coorpromazina) & - & $378 \mathrm{mg} / \mathrm{d}$ \\
\hline
\end{tabular}

\section{Medidas}

Test de Factor "g" (Cattell \& Cattell, 1990): prueba libre de influencias culturales que, en su versión española, se utiliza para evaluar la inteligencia individual.

Escala del síndrome positivo y negativo en la esquizofrenia (PANSS) (Kay, Fiszbein y Opler, 1987. Traducción de Peralta y Cuesta, 1994): entrevista semi-estructurada que evalúa tanto los signos negativos como positivos y la psicopatología global.

Prueba de verbos mentales: el constructo de TM se mide con una prueba extensiva de verbos mentales similar a la utilizada por Schwanenflugel et al., (1994 b). Consta de 28 frases, la mitad son afirmativas y la otra mitad interrogativas, que han de ser completadas con al menos tres de los verbos mentales de los 14 que se presentan como posibles respuestas; de modo que, posteriormente, esta elección mínima de tres verbos nos permitirá analizar las proximidades existentes entre las actividades mentales. Para seleccionar los verbos el sujeto ha de escoger los que mejor se ajusten a cada frase, bien porque al sustituir un verbo por otro no cambie el sentido de la frase, bien porque complete la oración, o bien porque responda a la pregunta que se plantea. Uno de los items de los que se compone la prueba fue: (COMPLETA) No sé dónde he dejado el paraguas y lo tengo que...: adivinar, aprender, atender, buscar, comparar, creer, decidir, elegir, entender, escuchar, mirar, pensar, recordar y saber 


\section{Procedimiento}

El Grupo Clínico cumplimentó individualmente y en varias sesiones las pruebas: Test de factor " $g$ ", Escala del síndrome positivo y negativo en la esquizofrenia (PANSS) y Tarea extensiva de verbos mentales. Por su parte, al Grupo Control se le aplicó colectivamente el Test de factor " $g$ " y la Prueba de verbos mentales.

\section{Análisis Estadísticos}

Para examinar la organización y el sentido que tanto el Grupo Clínico como el Grupo Control dan a los verbos mentales se utilizó el escalamiento multidimensional. Dicho análisis favorece la construcción de un espacio métrico con el menor número de dimensiones posibles que faculta para representar proximidades o preferencias entre objetos. Los datos de entrada se entienden como estimaciones subjetivas de la proximidad entre estímulos por lo que se obtiene una distribución en un espacio de varias dimensiones, de tal forma, que las distancias entre los estímulos en ese espacio se corresponderían con las proximidades expresadas subjetivamente; pero además, se extraerian una serie de ejes (uno por dimensión), cada uno de los cuales representaría la escala o atributo que ha manejado el sujeto al estimar las proximidades, así como el lugar de los estímulos en cada una de esas escalas en forma de coordenadas en el eje correspondiente (Real, 2001). El número de dimensiones a conservar suele determinarse teniendo en cuenta algún criterio estadístico que mida la bondad de ajuste de los datos a la hora de aceptar o rechazar la configuración de puntos obtenida; el S-stress y el stress siven a tal efecto, ya que cuanto menor es el stress, mejor es el ajuste. Pero, no hay que olvidar otros aspectos como la facilidad de interpretación y el uso de los resultados, siempre teniendo presente que los puntos de una agrupación se conectan perfectamente entre ellos y mal con los que están fuera de la mencionada agrupación (Bisquerra, 1989; Kruskal y Wish, 1984).

El área más sugerente para aplicar este tipo de análisis, es la del desarrollo cognitivo en la que se ha intentado mostrar que los niños pequeños organizan los estímulos a lo largo de dimensiones 
perceptivas, mientras que los mayores emplean unas dimensiones más vagas y complejas por tratarse de dimensiones conceptuales e internas; parece, por lo tanto, que con la edad se incrementa la importancia dada a las dimensiones más abstractas. También, el escalamiento multidimensional es muy útil en la comprensión de los efectos del contexto al mostrarnos como los pesos de las dimensiones varían, aparte de con la edad, en función del nombrado contexto lo que genera un cambio en la estructura de los datos (Shoben y Ross, 1987).

\section{RESULTADOS Y CONCLUSIONES}

\section{Fase A: Designación y utilización de los verbos mentales por parte del Grupo Control}

Para estos sujetos se obtienen las siguientes medidas de ajuste y estrés: estrés bruto normalizado 0.0789 , dispersión explicada 0.921 y coeficiente de congruencia de Tucker 0.9597.

En el Gráfico 1, y en función de la selección realizada por el mencionado grupo cuando cumplimenta la tarea extensiva de verbos mentales, aparecen las representaciones espaciales de los estimulos verbales. En ellas se aprecia que tales actividades mentales se agrupan en las categorias de:

Input (I): compuesta por los verbos atender, escuchar; por tanto, falta mirar y se añade entender (General -G-).

Memoria (M): formada por los verbos recordar y saber; asimismo, y en esta categoría, se introduce aprender (G).

Procesamiento $(P)$ : compuesta por adivinar, comparar, creer, decidir, elegir y pensar (creer y adivinar se introducen en esta agrupación debido a que el punto máximo de inclusión establecido es 0.03). También se añaden los verbos mirar (I), buscar (G).

La lejania nos muestra que son mirar (I) y pensar $(P)$, destacados con ", los verbos más alejados, bien con respecto a las representaciones espaciales de todos los verbos, como en el primer caso, bien con respecto al grupo al que pertenece, en el caso del verbo pensar (P).

En cuanto a la ubicación de los estímulos en los ejes correspondientes de abscisas (dimensión 1 -D1-: certeza-duda) y ordenadas (dimensión 2 -D2-: interno/conceptual - externo/ perceptivo) 
observamos, que, al nombrar los distintos cuadrantes en el sentido de las agujas del reloj, las tres categorias de verbos mentales quedan distribuidas como a continuación se indica:

I: los verbos que la componen se colocan en el segundo cuadrante.

M: sus actividades mentales se centran en el primer cuadrante.

P: la situación de esta agrupación corresponde sobre todo al cuarto cuadrante, aunque también aparece algún verbo en el tercero, concretamente elegir.

Gráfico 1. Representaclón espacial de los verbos mentales correspondiente al grupo control

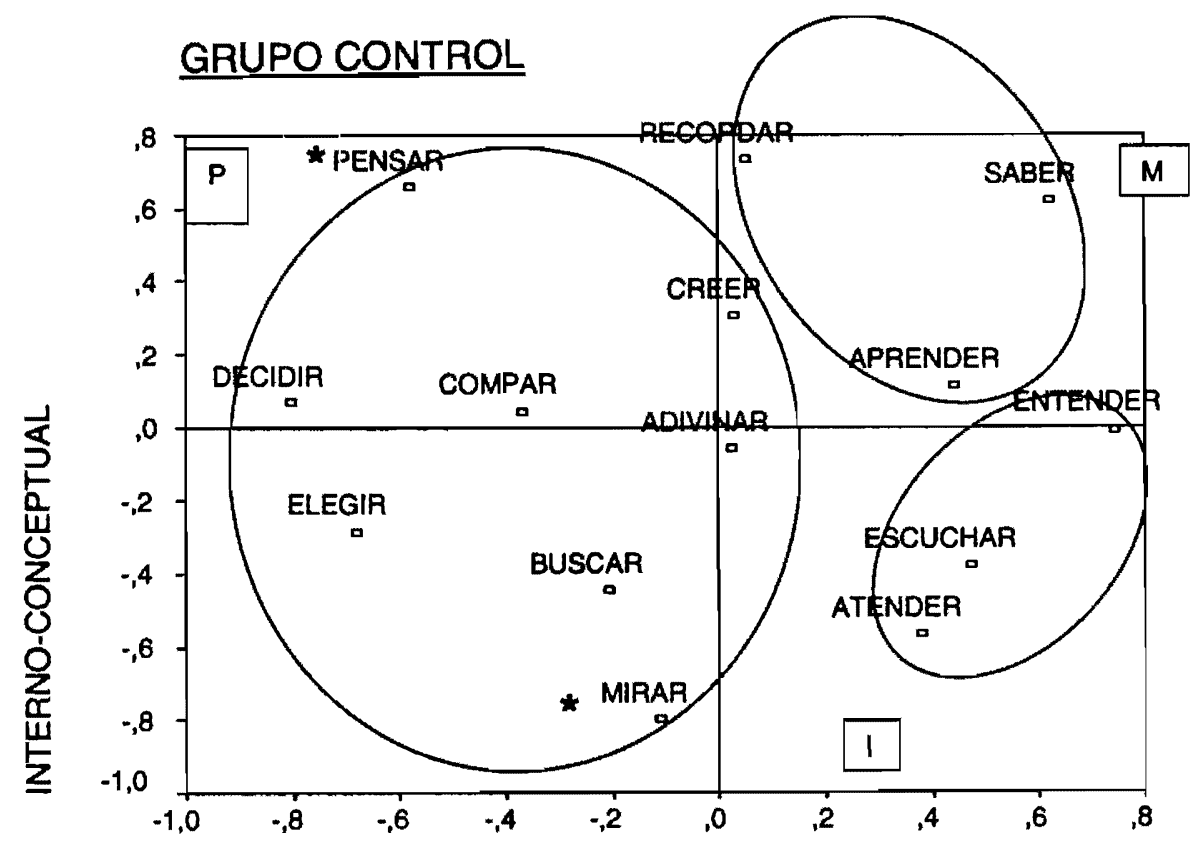

CERTEZA

Por tanto, diremos que en este grupo aparecen las tres categorías de verbos mentales, esto es: I, M y P. Las agrupaciones de $M$ y $P$ están completas, es decir, compuestas por todos los verbos que las forman; sin embargo, en la categoría de l encontramos que falta el verbo mirar, actividad que se introduce en $P$, lo cual no resulta extraño si, tal y como Schwanenflugel y sus colaboradores plantean, dicha actividad tiene un significado distinto al otorgado al verbo "ver", definiéndose como 
un verbo más dudoso y así, cercano a la categoría de procesamiento. También se observan una serie de actividades mentales que determinan la agrupación denominada General (G) con verbos muy versátiles que forman parte de otras categorías $y$, ni se definen en función de una concreta, ni se agrupan formando una propia. De este modo, su perfil de uso es muy amplio, pero, correcto. En este caso aparecen en I entender (G), en $M$ aprender (G), y en $P$ buscar ( $G)$.

Al analizar la lejanía hallamos que mirar (I) es el más alejado en el conglomerado de la representación espacial de los datos y ello como consecuencia de las características que comparte con los verbos de procesamiento. Asimismo, pensar, aunque forma parte de la categoría de procesamiento, es el más distante, debido a la función evaluadora de este verbo, pues pensar es una actividad cognitiva muy común que forma parte de diversos procesos cognitivos (Rips y Conrad, 1989; Schwanenflugel et al., 1994b, 1998).

Si se parte de la posición de los estímulos verbales en las dos dimensiones establecidas por Clark et al. (1996), Moore et al. (1990) y Schwanenflugel et al. (1996, 1998, 1999): dimensión 1: certeza- duda; dimensión 2: interna y conceptual - externa y perceptiva), encontramos que los verbos de I se definen como externos, perceptivos y ciertos; situándose en el cuadrante segundo. Los pertenecientes a la categoría de $\mathrm{M}$ son internos, conceptuales y ciertos, apareciendo en el primer cuadrante. Los verbos de $\mathrm{P}$ están prioritariamente en el cuarto cuadrante con un significado de verbos mentales dudosos, internos y conceptuales, excepto el verbo elegir, que aparece en el tercer cuadrante y se determina como externo y perceptivo.

De los datos expuestos deducimos que el Grupo Control asigna significados específicos a los verbos de actividad mental, lo que se refleja en la clara definición, diferenciación y formación de las categorlas de l, $M$ y $P$. En cuanto a las actividades que abarca la categoría de $G$ tienen un perfil de uso más extenso $y$, asi, pueden aparecer ubicados en categorias distintas a la propia, sin que ello perjudique o contamine a las categorías de destino en las que se incluyen. Por ello, la utilización de los verbos mentales realizada por este grupo será muy correcta y madura y su comunicación no presentará ningún déficit, pues no olvidan que sus conocimientos, necesidades, intenciones y deseos pueden ser diferentes y mostrarse de modo distinto a los de otras personas. Asl, a 
partir de ahora nosotros nos basaremos en los resultados y conclusiones obtenidos en el Grupo Control para analizar los alcanzados en la Fase $B$ de este estudio.

Fase B: Designaclón y utllización de las actividades mentales por parte del Grupo Clínico

Para analizar la sintomatología que presentaban los sujetos se ha utilizado la Escala del síndrome positivo y negativo en la esquizofrenia (PANSS) (Kay et al., 1987). Los resultados obtenidos en tal escala y, detallados en la Tabla 2, han fragmentado al grupo clínico en: SIGNGAT 0 o grupo de enfermos que no presentan signos negativos, SIGNGAT 1 compuesto por pacientes que tienen signos negativos, SIGPOTV 0

Tabla 2. Resultados del grupo clínlco en PANSS

\begin{tabular}{|c|c|}
\hline Sujeto & PANSS \\
\hline 1 & 6 \\
\hline 2 & 1.2 \\
\hline 3 & $1-2-3$ \\
\hline 4 & 2 \\
\hline 5 & $3-5$ \\
\hline 6 & 2 \\
\hline 7 & 5 \\
\hline 8 & 2 \\
\hline 9 & 5 \\
\hline 10 & $1-2$ \\
\hline 11 & 5 \\
\hline 12 & 2 \\
\hline 13 & $3-4$ \\
\hline 14 & $1-2.5$ \\
\hline 15 & $2-3-4$ \\
\hline 16 & $1-2-4-5$ \\
\hline 17 & 2 \\
\hline 18 & $2-5$ \\
\hline 19 & $1-3$ \\
\hline 20 & 1.2 \\
\hline 21 & $2-3-4$ \\
\hline 22 & $1-2-5$ \\
\hline 23 & 2 \\
\hline 24 & $2-5$ \\
\hline 25 & $2-3-4-5$ \\
\hline 26 & $2-3-4-5$ \\
\hline 27 & $1-2-3-5$ \\
\hline
\end{tabular}


formado por esquizofrénicos que no presentan signos positivos, SIGPOTV 1 ó grupo de esquizofrénicos que presentan signos positivos, SIGNGATPOTV 1 esquizofrénicos que tienen signos negativos y positivos, SINPARA 0 enfermos sin síntomas paranoides, SINPARA 1 sujetos con síntomas paranoides, SINPASI 0 pacientes sin síntomas de pasividad, SINPASI 1 grupo de esquizofrénicos con síntomas de pasividad, y OTROSSREM 1 PUROS compuesto por aquellas personas que sólo tienen otros síntomas menos típicos o que están en remisión.

SIGNGAT 0 obtiene un estrés bruto normalizado de 0.0853 , una dispersión explicada de 0.9146 y un coeficiente de congruencia de Tucker de 0.9563. Dada la selección de las actividades mentales realizada por este grupo cuando cumplimenta la tarea extensiva de verbos mentales, las diferentes categorías mentales se definen con los verbos que se detallan a continuación (ver Gráfico 2):

I: atender, escuchar, falta mirar y se introducen aprender/entender $(G)$.

M: no forma grupo.

$P$ : adivinar, comparar, decidir, elegir y pensar, falta creer e incluyen buscar (G), mirar (I), recordar (M).

La lejania o no proximidad se centra en pensar $(P)$.

La ubicación de los estímulos en los ejes de coordenadas nos permite concluir que las categorías están:

I: cuadrante segundo.

P: cuadrantes tercero y cuarto.

SIGNGAT 1 con un estrés bruto normalizado de 0.0897 , una dispersión explicada de 0.9102 y un coeficiente de congruencia de Tucker de 0.954 distribuye los verbos mentales en función de las categorías (Gráfico 3):

I: atender, escuchar, mirar, así como adivinar (P)

M: no forma grupo.

$P$ : comparar, creer, decidir, elegir y pensar; falta adivinar y también, se añaden buscar $(G)$, recordar $(M)$.

La distancia se observa en mirar $(I)$ y pensar $(P)$.

Las categorías de actividades mentales se distribuyen en relación a los ejes de abscisas y orcenadas del modo siguiente:

I: cuadrante tercero.

P: cuadrantes primero y segundo.

De tal forma que en el estudio de los signos negativos destacamos que el grupo SIGNGAT 0 (esquizofrénicos sin signos negativos) sólo 
Gráfico 2. Representación espacial de los verbos mentales correspondiente al grupo que no presenta signos negativos

\section{SIGNGAT 0}

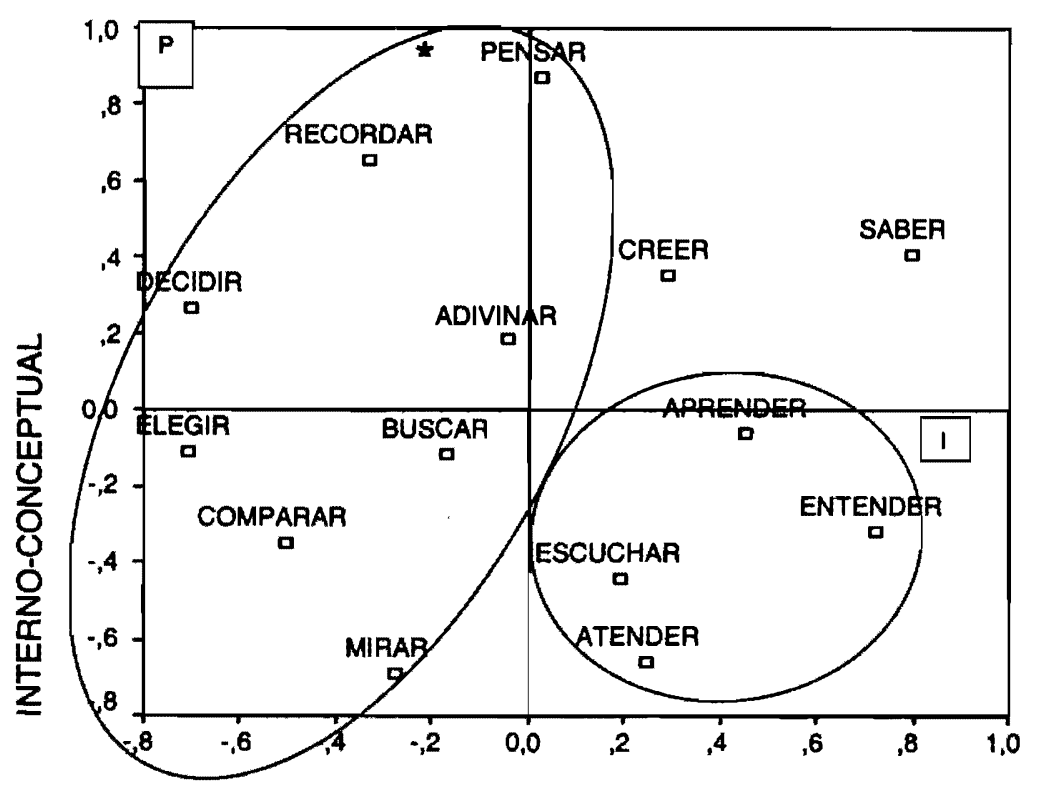

CERTEZA

determina las categorías de 1 y de $P$, aunque en esta última falta creer y se añade un verbo tan inusual como recordar (M). SIGNGAT 1 (con signos negativos) tampoco define la categoría de $\mathrm{M}$, la agrupación de I recibe la contaminación de adivinar $(P)$ y la categoría de $P$ aparece incompleta, ya que falta adivinar, y se introduce recordar(M). La lejanía recae en los verbos usuales mirar $(I)$ y pensar $(P)$. $Y$ en relación a las dimensiones de certeza-duda e interna y conceptual-externa y perceptiva se refiere, las categorias se determinan del modo siguiente: en SIGNGAT O la agrupación de I se presenta como externa, perceptiva y cierta y la de $P$, dudosa, interna y conceptual, exceptuando comparar y elegir, definidos como externos y perceptivos. En SIGNGAT 1 la agrupación de les externa, perceptiva, pero dudosa y la de P, cierta, externa y perceptiva, salvo creer y pensar, que se muestran más internos y conceptuales. 
Gráfico 3. Representación espacial de los verbos mentales correspondiente al grupo que presenta signos negativos

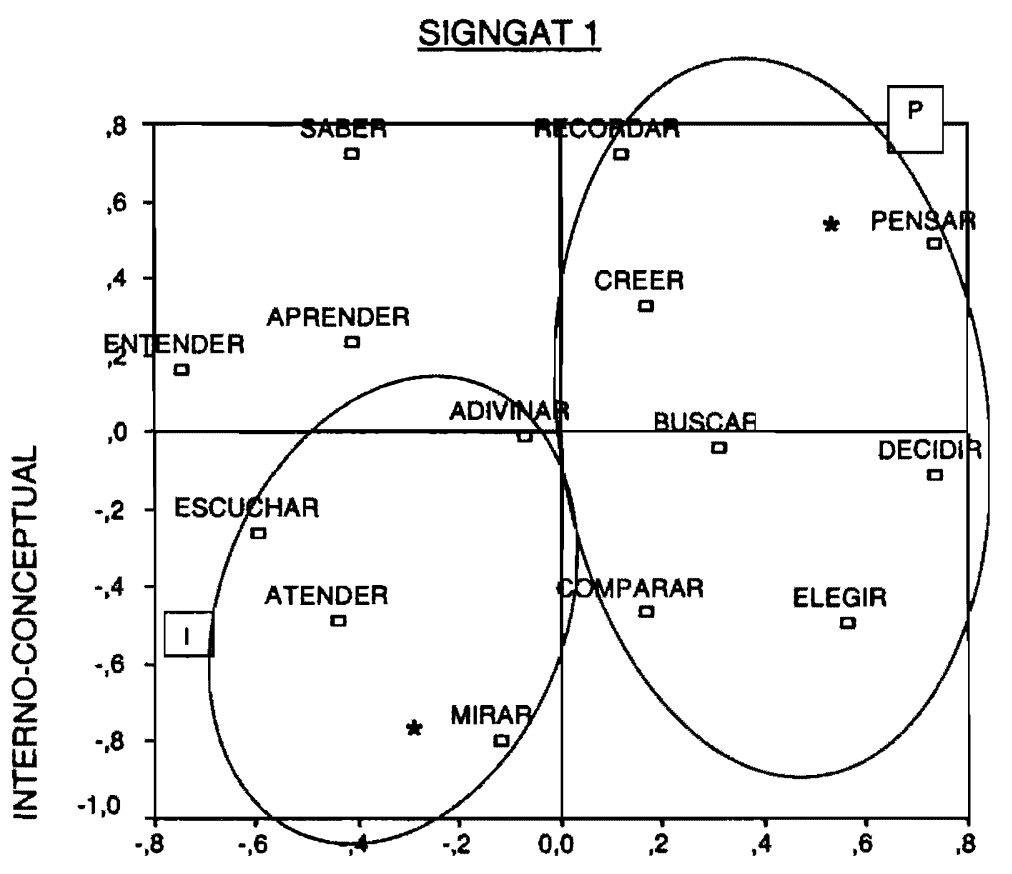

CERTEZA

No se observan destacadas diferencias entre los conjuntos de enfermos sin y con signos negativos. Las categorias de verbos mentales se forman y definen de modo similar, salvo en la agrupación de I, que en SIGNGAT 1 está más contaminada al incluir adivinar $(P)$. Pero, la ubicación realizada por SIGNGAT 0 es la que mejor se ajusta a la hallada en el Grupo Control. SIGNGAT 0 y el Grupo Control coinciden en que I es una categoría externa, perceptiva y cierta y $P$, una categoría de más duda, interna y conceptual, exceptuando los casos de comparar y elegir, vistos como externos y perceptivos.

Siguiendo a Corcoran y Frith (1996) y Frith y Corcoran (1996), diremos que los pacientes con síntomas negativos muestran serios déficits en TM. En concreto, en el reconocimiento de intenciones veladas, de falsas creencias, una tendencia a no usar en sus 
explicaciones un lenguaje centrado en los estados mentales. Aunque, Corcoran (2001), Doody et al. (1998), González, Alonso del Teso, Mínguez y Sanguino (2003) y Murray, $\mathrm{O}^{\circ}$ Callaghan, Castle y Lewis (1992) encuentran que los signos conductuales negativos se asocian con una pobre realización de las tareas de falsas creencias porque podrian reflejar unos déficits cognitivos generales relacionados con dichas características negativas, con un pobre almacenamiento de la información relevante, con la cronicidad, con el comienzo precoz de la enfermedad y con una limitada experiencia social. Y quizás sea por estos motivos por los que la distribución de las categorías en las dos dimensiones no se ajusta completamente a la del Grupo Control, aunque es tendente a ella. De modo que pese a la gran influencia que la presencia de los signos negativos ejerce en la resolución de la tarea de verbos mentales que genera que la distribución, denominación y categorización de los verbos mentales sea la peor de todas las observadas (como veremos al examinar el resto de la sintomatología), la no presencia de tales signos también depende de otros factores, de ahí la incorrección observada en la tarea pese al no efecto de dichos síntomas. Pero, sí hemos de decir que se esperaban unas diferencias más importantes entre ambos grupos (sin y con signos negativos), diferencias que bien pudiera ser que no se muestren debido al efecto compensador de otros factores (por ejemplo del cociente intelectual).

SIGPOTV 0 obtiene un estrés bruto normalizado 0.0878 , una dispersión explicada 0.9121 y un coeficiente de congruencia de Tucker 0.955 .

En el Gráfico 4 aparecen las categorias de verbos mentales y los verbos que las componen:

I: atender, escuchar; falta mirar (I) y se introducen aprender/entender (G).

M: recordar y saber.

$P$ : adivinar, comparar, creer, decidir, elegir y pensar; se añaden buscar (G), mirar (I).

La lejania implica a los verbos entender $(G)$, mirar $(I)$ y pensar $(P)$.

En un espacio definido por las dos dimensiones observamos que los estímulos verbales de las diferentes categorías estarían en:

l: cuadrante segundo.

M: cuadrante primero.

$P$ : cuadrantes tercero y cuarto.

SIGPOTV 1 obtiene un estrés bruto normalizado de 0.0966 , una 
dispersión explicada de 0.9033 y un coeficiente de congruencia de Tucker de 0.9504.

Las categorias de verbos mentales en las que se distribuyen tales actividades son las siguientes (ver Gráfico 5):

I: atender, escuchar y mirar; se añade, también, comparar (P).

$M$ : recordar y saber; y se introducen entender $(G)$ y adivinar $(P)$.

$P$ : creer, decidir, elegir y pensar; faltan adivinar y comparar. Asimismo, se introducen aprender/buscar (G).

La mayor distancia recae en pensar $(P)$ y saber $(M)$.

En los ejes de coordenadas las categorias de verbos mentales se ubican en:

I: cuadrante tercero.

M: cuadrante cuarto.

$P$ : cuadrantes primero y segundo.

Así, al analizar la influencia de los signos positivos hallamos que SIGPOTV 0 (sin signos positivos) reconoce la existencia de las tres

Gráfico 4. Representación espacial de los verbos mentales correspondiente al grupo que no presenta slgnos positivos

\section{SIGPOTVO}

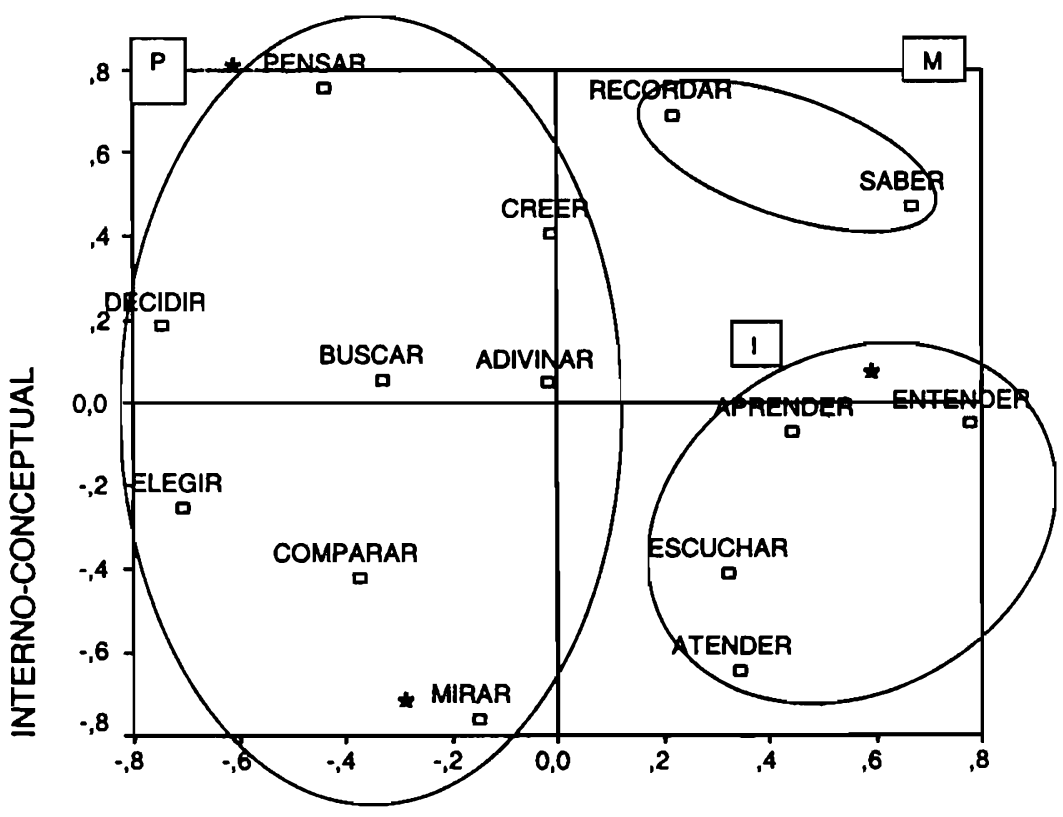

CERTEZA 
categorias que están bastante definidas y de un modo semejante a como lo hace el Grupo Control. En SIGPOTV 1 (con signos positivos) aparecen ciertas novedades, de modo que las tres categorias aparecen menos detalladas. Así, en I se añade comparar ( $P$ ), en $M$ adivinar ( $P$ ) y en $P$ faltan adivinary comparar. La distancia en SIGPOTV 1 implica a un verbo extraño, saber (M). Pero es la representación espacial de los verbos en las dimensiones la que muestra más datos relevantes. Por un lado, SIGPOTV 0 define I como externa, perceptiva y cierta, M como interna, conceptual y cierta y $P$ como dudosa, interna y conceptual, menos para comparar y elegir, vistos como externos y perceptivos. Por otro lado, SIGPOTV 1 detalla I como externa, perceptiva y dudosa, $\mathrm{M}$ como interna, conceptual y dudosa, y $\mathrm{P}$ como cierta, interna y conceptual, excepto en los casos de decidir y elegir, entendidos como externos y perceptivos.

SIGPOTV 0 determina y compone mejor las categorías verbales sin permitir la intrusión de verbos ajenos e inusuales, sólo los pertenecientes a la categoría $G$, debido a su gran adaptabilidad, y mirar

Gráfico 5. Representaclón espacial de los verbos mentales correspondlente al grupo que presenta signos positivos
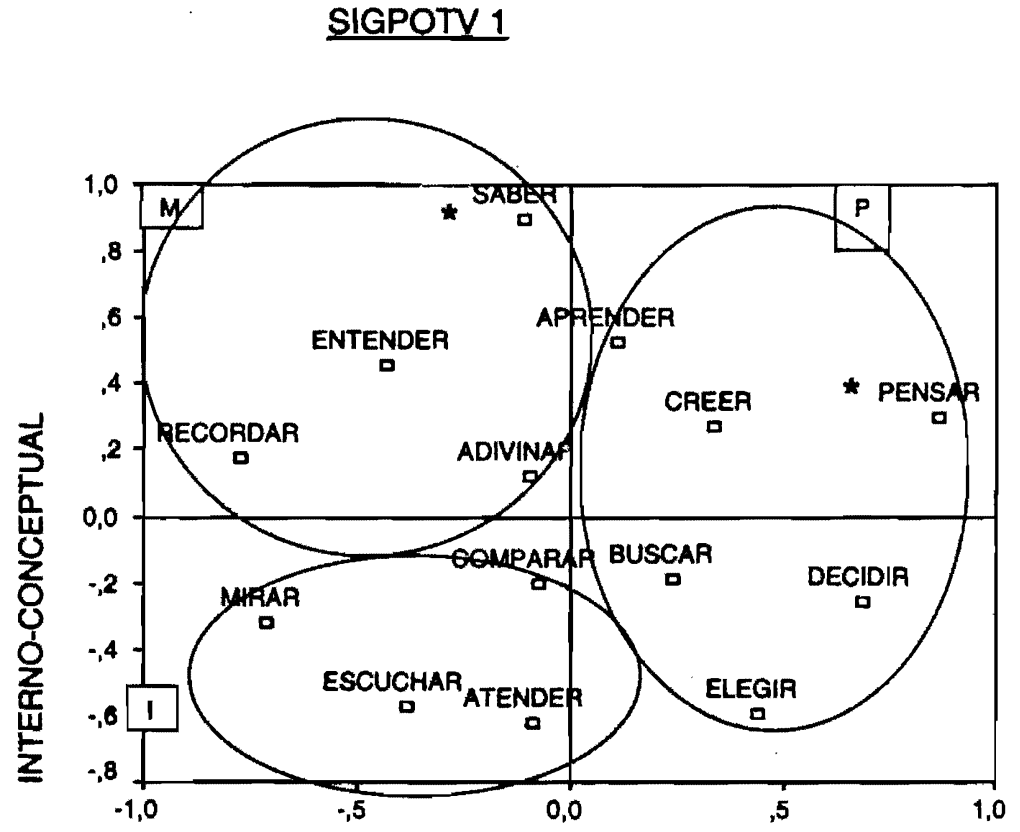

CERTEZA 
(I), como verbo cercano a la categoría de procesamiento. La no proximidad es más restringida en SIGPOTV 0 que en SIGPOTV 1. La ubicación de las actividades mentales en las dos dimensiones que mejor se ajusta a la realizada por los controles es la realizada por SIGPOTV 0.

Tal y como argumentan Corcoran (2001), Sarfati (2000), Sarfati y Hardy- Baylé (1999) y Sarfati, Hardy-Baylé, Besche, Widlöcher (1997 a), Sarfati et al. (1997 b), Sarfati, Hardy-Baylé, Brunet y Widlöcher (1999) apoyamos la idea de que los esquizofrénicos con desorganización del pensamiento y trastornos del lenguaje presentan dificultades significativas en la atribución de estados mentales a otras personas, dado que estos enfermos aparentemente no reconocen la diferencia entre su estado de conocimiento y el del oyente. Nuestros resultados son coherentes con estas observaciones en el sentido de que los fallos detectados en SIGPOTV 1 se manifiestan también en una errónea comprensión y expresión de los verbos de actividad mental y por tanto, en una limitada comunicación. La enorme semejanza observada entre SIGPOTV 0 y el Grupo Control la interpretamos en función de la gran influencia que la no presencia de los signos positivos tiene en la resolución adecuada de la tarea propuesta.

SIGNGATPOTV 1 obtiene un estrés bruto normalizado de 0.0901 , una dispersión explicada de 0.909 y un coeficiente de congruencia de Tucker de 0.9538 .

Las distintas categorias de actividades mentales son y están formadas por (ver Gráfico 6):

I: atender, escuchar, mirar; y también adivinar $(P)$.

M: no forma grupo.

$P$ : comparar, creer, decidir, elegir y pensar; no aparece adivinar y se añaden buscar $(G)$, recordar $(M)$.

La no proximidad recae, en este caso, en mirar (I) y pensar (P).

Los ejes de coordenadas nos ayudan a definir las distintas categorías de actividades mentales en:

I: cuadrante tercero.

P: cuadrantes primero y segundo.

De este modo, al agrupar los sujetos que presentan tanto signos negativos como signos positivos, hallamos que SIGNGATPOTV 1 sólo define las categorias de I y $P$, aunque en la primera se introduce adivinar 
(P), y es este verbo el que falta en $P$, categoria en la que también se añade recordar (M). La lejanía afecta a las actividades acostumbradas. Y la distribución de los estímulos en las dos dimensiones nos ofrece que I se destaca como externa, perceptiva y dudosa, mientras que $P$ es cierta, externa y perceptiva, salvo creery pensar, que se aprecian como más internos y conceptuales. Al igual que Allen et al. (1993), Corcoran (2001), Frith et al. (1991), resaltamos que los pacientes con cualquier signo conductual negativo o positivo obtienen puntuaciones bajas en tareas de TM, ya que no pueden representar los estados mentales y que la pobreza e incoherencia en el discurso, el afecto aplanado e incongruente y el retardo psicomotor se asocian a un déficit cognitivo, independiente de los efectos de las características negativas.

SINPARA 0 el estrés bruto normalizado es de 0.0845 , la dispersión explicada de 0.9154 y el coeficiente de congruencia de Tucker de 0.9568 .

Gráfico 6. Representación espacial de los verbos mentales correspondiente al grupo que presenta signos negativas y positivos

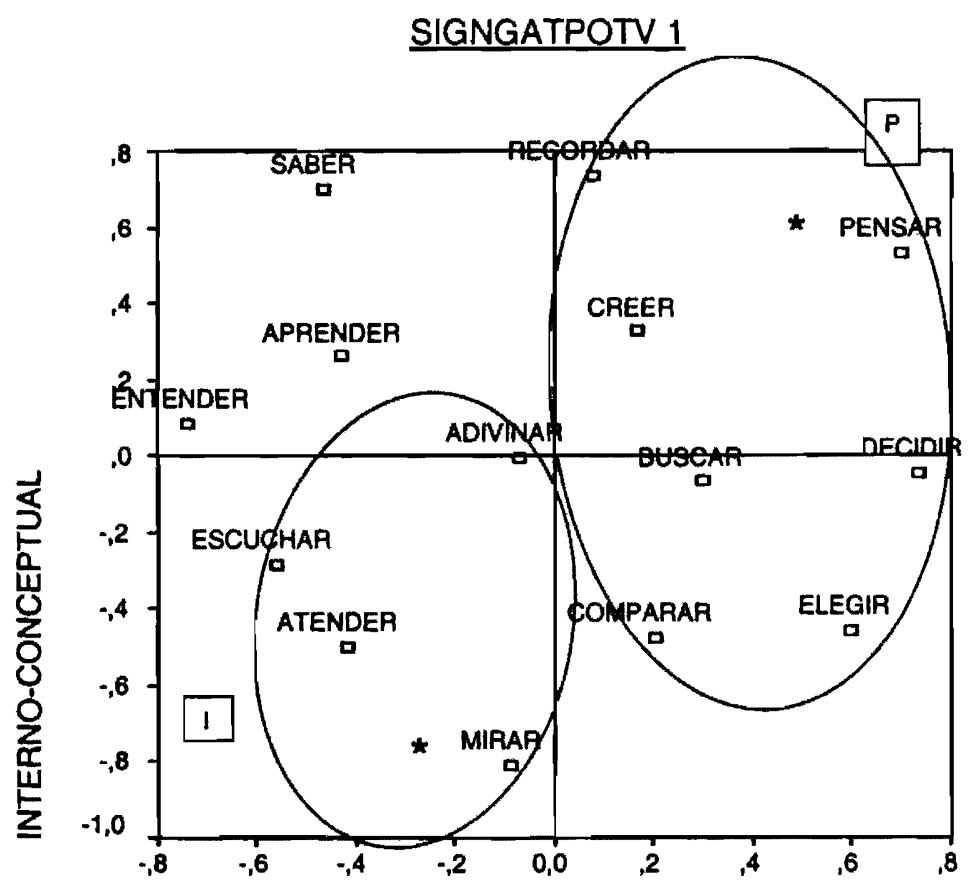

CERTEZA 
Las categorías que en esta agrupación se observan están formadas por (ver Gráfico 7):

I: atender, escuchar, mirar.

M: no forma grupo.

$P$ : adivinar, comparar, decidir, elegir y pensar, - falta creer y se introducen buscar $(G)$, recordar $(M)$.

La lejanía se aprecia en entender $(G)$, mirar (l) y pensar $(P)$.

La distribución espacial en función de las dos dimensiones establecidas nos revela que los verbos mentales están situados en:

I: cuadrante tercero.

P: cuadrantes primero y segundo.

SINPARA 1 obtenemos un estrés bruto normalizado 0.0902 , una dispersión explicada 0.9098 y un coeficiente de congruencia de Tucker 0.9538 .

En el Gráfico 8 se aprecian la distribución y composición de las categorias de verbos:

I: atender, escuchar; falta mirar y se introduce recordar (M).

M: no forma grupo.

$P$ : adivinar, creer, decidir, elegir; faltan comparar y pensar.

La no proximidad recae en pensar (P) y mirar (I).

La distribución de los verbos mentales en función de los ejes de abscisas y ordenadas se centran en:

l: cuadrante segundo.

P: cuadrante cuarto.

Como conclusiones diremos que SINPARA 0 (sin síntomas paranoides) sólo define las agrupaciones de I y $P$, aunque en esta última falta creer y se añade recordar (M) y que en SINPARA 1 (con síntomas paranoides) en I se añade recordar $(M)$, no aparece $M$ y en $P$ faltan comparary pensar. La lejania se centra en verbos de $G$, mirar (I) y pensar (P). En cuanto a la ubicación de los estímulos verbales en las dos dimensiones SINPARA 0 entiende que I es externa, perceptiva y dudosa y $P$ cierta, externa y perceptiva, salvo adivinary pensar, que se muestran como más internos y conceptuales. En SINPARA 1 la categoría de I es externa, perceptiva y cierta y $P$, dudosa, interna y conceptual.

En ambos grupos falta la categoria de $\mathrm{M}$, pero SINPARA 0 realiza una definición algo más clara y completa de las agrupaciones 
Gráfico 7. Representación espacial de los verbos mentales correspondiente al grupo que no presenta síntomas paranoldes

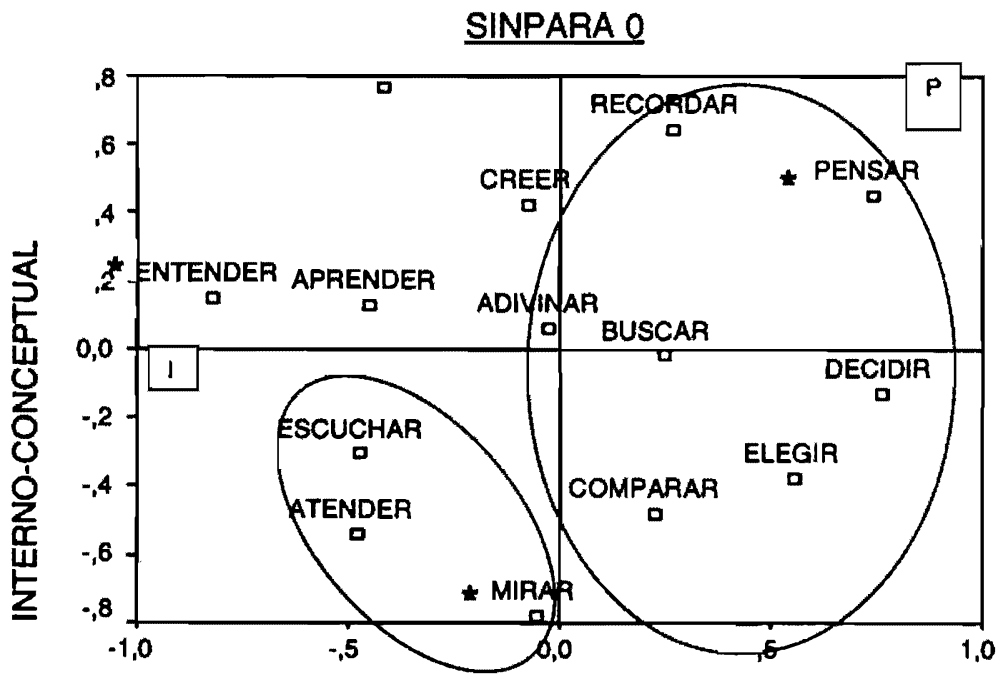

CERTEZA

correspondientes a I y P. Sin embargo, la ubicación de las actividades mentales en un espacio de dos dimensiones revela que es SINPARA 1 la agrupación que más se acerca a la disposición establecida por el Grupo Control en las categorías de input y procesamiento; no obstante, recordemos que l está contaminada por actividades no pertenecientes a la categoría y $\mathrm{P}$ incompleta. Así, nuestra postura es contraria a la de Herold, Tényi, Lénárd y Trixler (2002), Langdon et al. (1997) y Mitcheley et al. (1998), quienes no encuentran evidencias de que los síntomas paranoides fueran asociados a una dificultad en TM y que los pacientes con tales sintomas ejecutaran peor que los controles las tareas de falsas creencias.

No observamos una gran diferencia entre los dos conjuntos de enfermos, pues la mejor distribución de los verbos mentales en SINPARA 0 no se acompaña de una adecuada definición de las categorías en sus respectivos cuadrantes y, al revés, en SINPARA 1. Una interpretación se centraría en la injerencia conjunta que otros signos y síntomas ejercen en el desempeño de los esquizofrénicos, así como en un efecto comparativamente más amplio de los signos 


\section{Gráfico 8. Representaclón espaclal de los verbos mentales correspondiente al grupo que presenta síntomas paranoides}

\section{SINPARA 1}

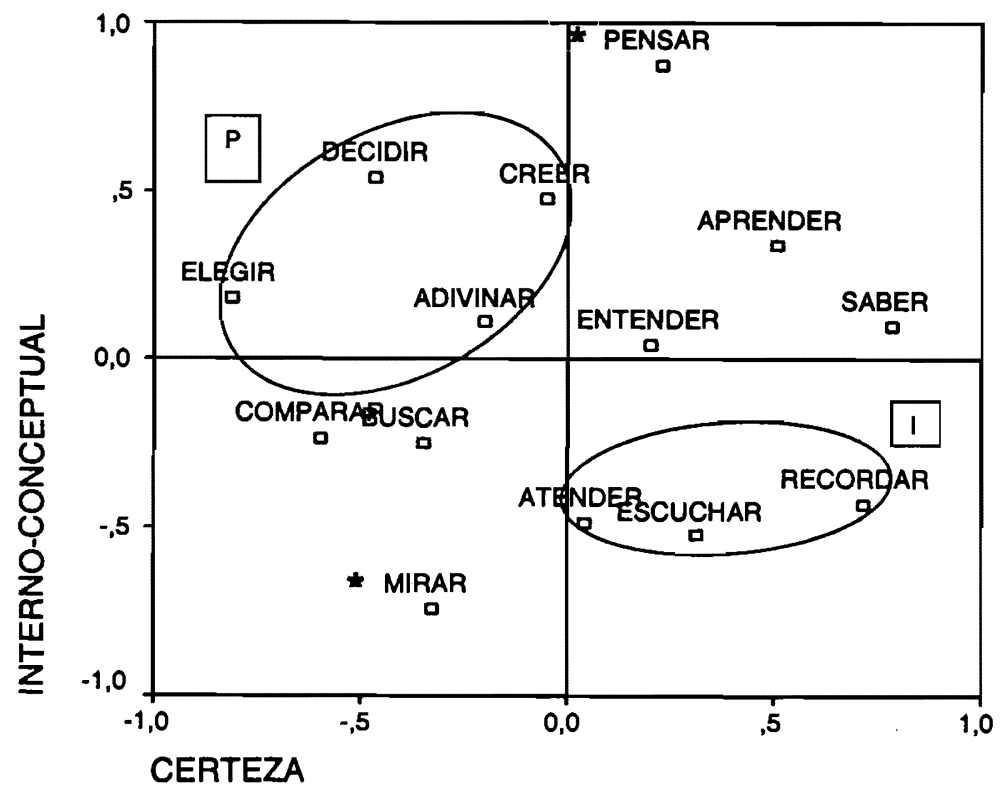

conductuales, como ocurre en muchos de nuestros pacientes (Corcoran, 2001 y Pickup y Frith, 2001). Otra se basaría en la adquisición y el desarrollo normal de la meta-representación de forma que los déficits sólo ocurren cuando comienza la enfermedad, por lo que estas personas aún tendrían habilidades residuales, incluso en estadios crónicos de la enfermedad, que se traducirian en una mejor realización de las tareas de TM. Sin embargo, ninguna de las agrupaciones realiza la tarea extensiva con total corrección, de manera que podemos entender que en este caso, incluso cuando no se experimentan síntomas paranoides, existen otros factores que median en la inadecuada solución de la tarea.

Si los resultados correspondientes a SINPARA 1 los asociamos con los obtenidos anteriormente por el grupo denominado SIGNGATPOTV 1, las diferencias observadas entre ellos, en cuanto a la formación de las categorías de I y $\mathrm{P}$ se refiere, son mínimas, pero en el análisis de las dos dimensiones establecidas, es donde, en SINPARA 1, aparece una definición más ajustada de ambas categorías. Por tanto, podemos 
afirmar que en el grupo SIGNGATPOTV 1 se exponen unos fallos más extensos en la tarea de verbos mentales que los mostrados por el grupo SINPARA 1, pues, tal y como valoran Corcoran y su equipo, mientras que los paranoides manifiestan problemas sólo en tareas complejas de segundo orden, los pacientes con signos conductuales tienen déficits más profundos en TM e, incluso, sus dificultades se plasman en las tareas de primer orden, en la comprensión pobre de las inferencias y en el desconocimiento de las reglas conversacionales. La explicación residiría en que las habilidades de TM, en los sujetos paranoides, están intactas hasta que comienza su enfermedad, durante la adolescencia o durante los primeros años de la vida adulta, por lo que tendrían ciertos restos, por ejemplo, centrados en las tareas de creencias falsas de primer orden, que les permitirian resolver con corrección este tipo de pruebas más sencillas.

SINPASI 0 obtiene un estrés bruto normalizado 0.0856 , una dispersión explicada 0.9143 y un coeficiente de congruencia de Tucker 0.9562.

En el Gráfico 9 se aprecia que los verbos mentales se agrupan asi: I: atender, escuchar y mirar.

$M$ : recordar y saber. Se introducen aprender/entender $(G)$.

$P$ : adivinar, comparar, creer, decidir, elegir y pensar. Se añade buscar (G).

La mayor distancia entre los verbos mentales se restringe a mirar (I) y pensar $(P)$.

La distribución en el espacio de dos dimensiones nos define las categorías de verbos mentales en:

I: cuadrante tercero.

M: cuadrante cuarto.

P: cuadrantes primero y segundo.

SINPASI 1 tiene un estrés bruto normalizado de 0.0977, una dispersión explicada de 0.902 y un coeficiente de congruencia de Tucker de 0.9498 .

En el Gráfico 10 se aprecian las distintas categorias en las que se distribuyen los verbos:

I: atender y escuchar; falta mirar. Se introducen aprender $(G)$, saber (M).

M: no forma grupo. 
$P$ : comparar, creer, decidir, elegir, faltan adivinar, pensar. Se incluyen buscar/entender (G) y mirar (I).

La lejanía recae en los verbos elegir/pensar (P) y mirar (I).

La ubicación espacial de los estímulos en los ejes revela que las categorías están en:

I: cuadrante tercero.

P: cuadrantes primero y segundo.

Gráfico 9. Representación espacial de los verbos mentales correspondiente al grupo que no presenta sintomas de pasividad

\section{SINPASIO}

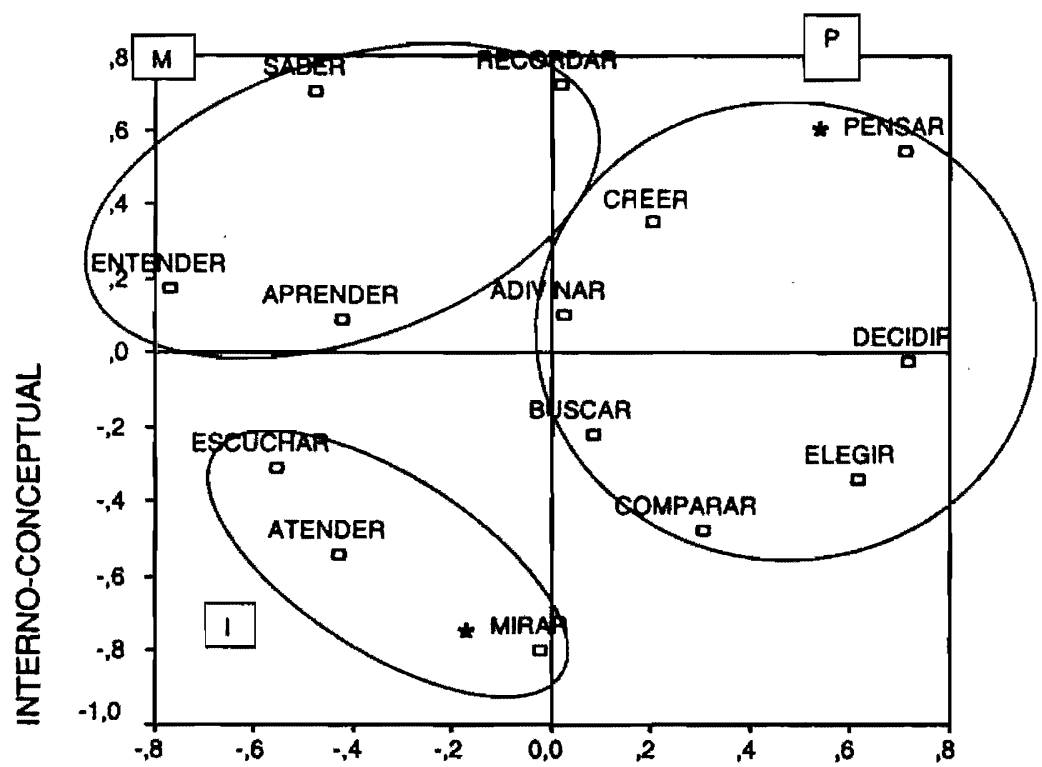

CERTEZA

Los síntomas de pasividad revelan que en SINPASI 0 (sin síntomas de pasividad) aparecen todas las categorías claramente definidas y formadas, mientras que en SINPASI 1 (con síntomas de pasividad) falta $M$, en I se introduce saber $(M)$ y en $P$ se omiten adivinar, pensar. La lejanía en SINPASI 0 se ciñe a las actividades conocidas (mirar, pensar), pero en SINPASI 1 es más extensa, implicando también a 
Gráfico 10. Representación espacial de los verbos mentales correspondiente al grupo que presenta sintomas de pasividad

SINPASI 1

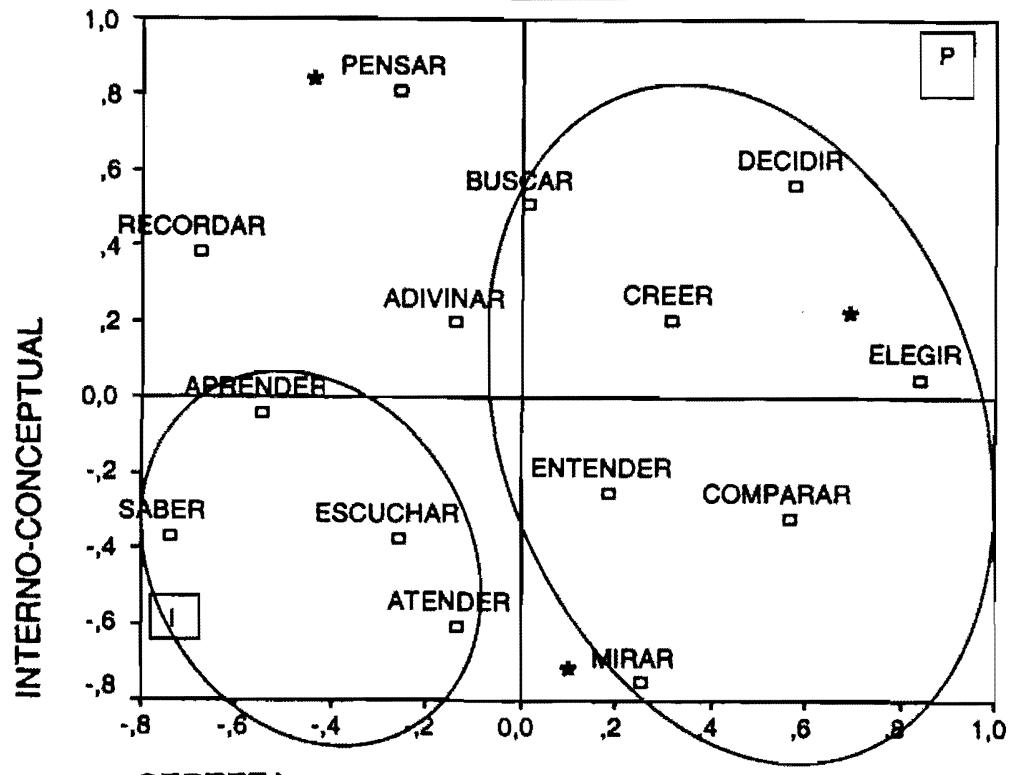

CERTEZA

elegir (P). Las dos dimensiones hacen que la distribución espacial de los verbos en sus categorías se detalle del modo siguiente: SINPASI 0 entiende que l es externa, perceptiva y dudosa, $M$, interna, conceptual y dudosa y $\mathrm{P}$, cierta, interna y conceptual, salvo comparary elegir, que se muestran como externos y perceptivos. De un modo bastante similar, SINPASI 1 determina I como externa, perceptiva y dudosa, y $P$, como cierta, interna y conceptual, aunque comparar se aprecia como más externo y perceptivo.

Es el grupo SINPASI 0 el que define e integra mejor las tres categorías de I, M y P. La distancia en SINPASI 1 es más extensa que en SINPASI 0 y la distribución de los estímulos verbales en las dos dimensiones hace que nos inclinemos por SINPASI 0 como el conjunto de sujetos que mejor define las categorías, pues al compararlo con el Grupo Control coinciden en especificar que: I es una categoría externa, perceptiva, $M$, interna y conceptual y $P$, interna y conceptual, excepto elegir, que se aprecia como externo y perceptivo (igual que ocurre en el Grupo Control), además de comparar. 
Por tanto, pese a que se esperaba que los pacientes con experiencias de pasividad realizaran las tareas de TM normalmente (Garety y Freeman, 1999), los resultados demuestran la influencia de los síntomas de pasividad en la realización de la prueba extensiva. Acaso, de nuevo, hemos de apoyarnos en Corcoran (2001), quien manifiesta que los resultados de los enfermos que dicen experimentar fenómenos de pasividad son confusos y que se desconoce hasta qué punto existe un efecto conjunto de otros signos y síntomas que afectan a la realización de las pruebas de TM.

OTROSSREM 1 PUROS con un estrés bruto normalizado 0.0806 , una dispersión explicada 0.9193 y un coeficiente de congruencia de Tucker 0.9588.

La distribución de los verbos mentales en las categorías sería (ver Gráfico 11):

I: atender, escuchar, falta mirar (I). Tarnbién aprender/entender (G).

$M$ : recordar y saber, se añade creer $(P)$.

Gráfico 11. Representación espacial de los verbos mentales correspondiente al grupo que presenta síntomas menos típicos o está en remislón

\section{OTROSSREM 1 PUROS}

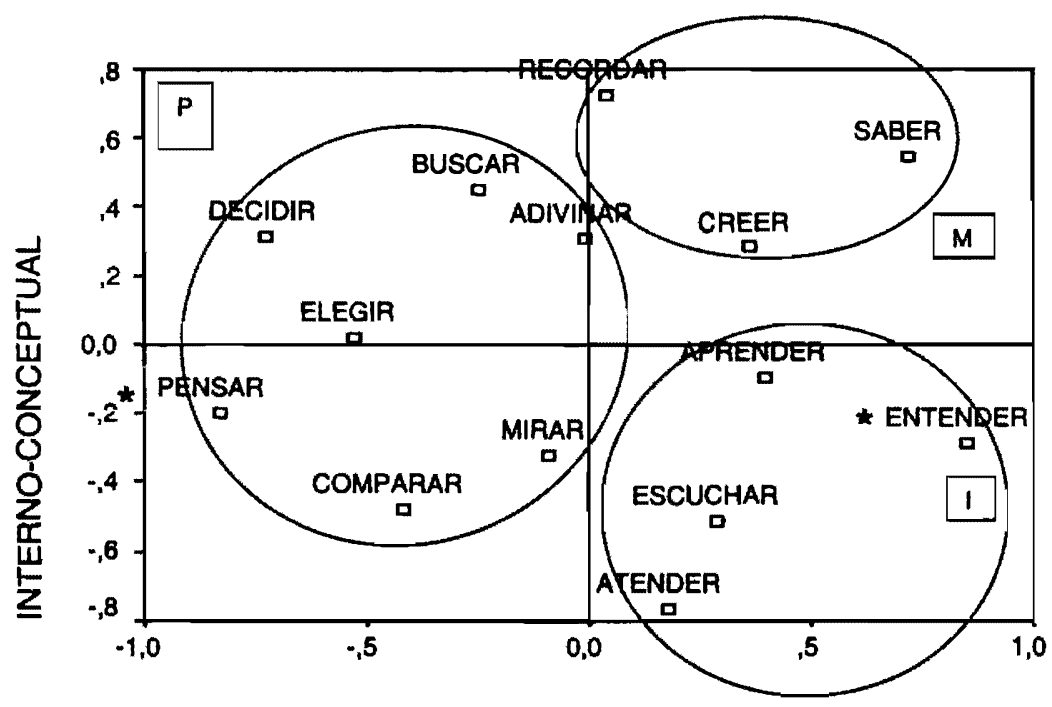

CERTEZA 
$P$ : adivinar, comparar, decidir, elegir y pensar; falta creer y se añade buscar (G), mirar (I).

La mayor distancia se concentra en entender $G$ ) y pensar $(P)$.

La ubicación de los estímulos en los ejes hace que las categorías estén en:

I: cuadrante segundo.

M: cuadrante primero.

$P$ : cuadrantes tercero y cuarto.

Manifestamos que en OTROSSREM 1 PUROS (con otros síntomas menos típicos o en remisión) aparecen las tres categorías de actividades mentales, sin embargo hay que destacar que en $M$ se introduce creer $(P)$, de forma que dicho verbo es el que se omite en $P$. La lejanía no nos sugiere datos relevantes al ceñirse a entender (G) y pensar $(P)$. $Y$ las dos dimensiones en las que se colocan los estímulos verbales hacen que la categoría de I se defina como externa, perceptivo y cierta, la categoría de $\mathrm{M}$ como interna, conceptual y cierta y $\mathrm{P}$, como dudosa, interna y conceptual, salvo en los casos de comparary pensar, vistos como externos y perceptivos.

Muy cercanas son las distribuciones encontradas en OTROSSREM 1 PUROS con respecto a las del Grupo Control, pues solamente en OTROSSREM 1 PUROS falta, en la categoría de P, creer, verbo que se añade a la categoría de $M$. En el resto de los aspectos evaluados, distancia y distribución en las dos dimensiones, no aparecen diferencias dignas de mención. Resaltamos, entonces, la afirmación de que en estos casos las habilidades en TM son normales y la solución de tareas de TM es tan correcta como la que realizan los grupos control no psicóticos. Asl, se concluye que la habilidad de mentalización es una variable estado, más que una variable rasgo, dependiente de los síntomas del paciente (Corcoran et al., 1995; Herold et al., 2002; Portela et al., 2003; Sarfati et al., 2000).

En resumen, los pacientes con Signos Negativos, Signos Positivos, Síntomas Paranoides y/o Síntomas de Pasividad no realizan la tarea de verbos mentales adecuadamente y su comunicación, especialmente cuando tienen que conversar sobre temas intangibles, como las falsas creencias y otros estados mentales abstractos, estará muy limitada. Por su parte, los esquizofrénicos con Otros Síntomas menos típicos o en Remisión solucionan casi perfectamente la prueba 
extensiva. Estos últimos grupos atribuyen significados específicos a los verbos mentales, diferencian con claridad las categorías de I, M y $P$ y participan adecuadamente en intercambios conversacionales que transmiten ideas o creencias abstractas.

Las agrupaciones SIGPOTV 0 y OTROSSREM 1 distribuyen las categorías de las actividades mentales en el espacio de un modo muy similar a como lo hace el Grupo Control, por lo que calificamos de una enorme trascendencia para la apropiada ejecución de la prueba extensiva el no presentar signos positivos, el tener otros síntomas menos comunes o el encontrarse en remisión. En el resto de los grupos, en los que se distribuyen los sujetos según su sintomatología, los datos manifiestan la intervención de otros factores y no sólo de los signos y síntomas en la resolución de la prueba de actividades mentales. Por ello, creemos conveniente examinar, también, los efectos e influencias en la resolución de tareas de TM: de los déficits cognitivos generales, del funcionamiento social, de la cronicidad y del inicio temprano de la enfermedad (Baddeley, Thornton, Chua y McKenna, 1996; Brüne, 2001; Corcoran, 2000, 2001; Corcoran et al., 1995; Fraser, King, thomas y Kendell, 1986; King, Fraser, Thomas y Kennedy, 1990; Langdon et al., 1997; Portela et al., 2003; Thomas et al., 1990).

En esta misma línea, seria muy provechoso investigar acerca de la relación existente entre la cronicidad y la sintomatología para intentar desvelar las sutiles diferencias encontradas entre la distribución y definición de las actividades mentales realizada por OTROSSREM 1 y la establecida por el Grupo Control (Drury et al., 1998; Sarfati et al., 2000).

Además, pensamos que es importante analizar el efecto conjunto de los signos y síntomas que median en la ejecución de las tareas de $T M$, en el sentido de que la sintomatología paranoide y los fenómenos de pasividad parecen verse superados por los efectos de los signos positivos y negativos, pero no tan ampliamente que conlleve la resolución errónea de tareas de primer orden.

No obstante hemos de decir que existen una serie de limitaciones en este estudio, como el diferente número de personas que forman las distintas agrupaciones y el solapamiento entre los sujetos de dichas agrupaciones, que nos alientan hacia la realización de investigaciones más profundas acerca de la influencia de los signos y síntomas de la esquizofrenia en la TM. 


\section{REFERENCIAS BIBLIOGRÁFICAS}

Allen, H., Liddle, P. y Frith, C.D. (1993). Negative features, retrieval processes and verbal fluency in schizophrenia. British Journal of Psychiatry, 163, 769-775.

Baddeley, A., Thornton, A., Chua, S.E. y McKenna, P. (1996). Schizophrenic delusions and the construction of autobiographical memory. En D.C. Rubin (Ed), Remembering our past: Studies in autobiographical memory. New York: Cambridge University Press.

Bisquerra, R. (1989). Introducción conceptual al análisis multivariable, vol. 1. Barcelona : PPU.

Bouchand, J. y Caron, J. (1999). Production de verbes mentaux et acquisition d'une theorie de l'esprit. Enfance, 51 (3), 225-237. Brüne, M (2001). Social Cognition and psychopathology in an evolutionary perspective. Psychopathology, 34, 85-94.

Cattell, R.B. y Cattell, A.K. (1990). Test de factor "g", escalas 2 y 3 (6 edición). Madrid: Tea Ediciones S.A. (Orig. 1973).

Clark, M.D., Schwanenflugel, P.J., Everhart, V.S. y Bartini, M. (1996).

Theory of mind in deaf adults and the organization of verbs of knowing. Journal of Deaf Studies and Deaf Education, 1 (3), 179189.

Corcoran, R. (2000). Theory of mind in other clinical conditions: is a selective "theory of mind" deficit exclusive to autism?. In S. BaronCohen, H. Tager-Flusberg y D. Cohen Understanding other minds (391-421) (2a edición). New York. Oxford University Press.

Corcoran, R. (2001). Theory of mind and schizophrenia. In P.W. Corrigan y D.L. Penn. Social cognition and schizophrenia (pp149174). Washington. American Psychological Association.

Corcoran, R, Cahill, C, y Frith, C.D. (1997). The appreciation of visual jokes in people with schizophrenia: a study of "mentalizing" ability. Schizophrenia Research 24, 319-327.

Corcoran, R y Frith, C. (1996). Conversational conduct and the symptoms of schizophrenia. Cognitive Neuropsychiatry, 1, 1105-1112.

Corcoran, R; Mercer, G y Frith, C.D. (1995). Schizophrenia, symptomatology and social inference: investigating theory of mind in people with schizophrenia. Schizophrenia Research, 17, 5-13. 
Doody, G.A, Götz,M., Johnstone,E.C., Frith,C.D. y Cunningham Owens, D.G. (1998). Theory of mind and psychoses. Psychological Medicine, 28, 397-405.

Drury, V.M, Robinson, E.J. y Birchwood, M. (1998). Theory of mind skills during an acute episode of psychosis and following recovery. Psychological Medicine, 28, 1101-1112.

Fraser, W, King, K, Thomas, P y Kendell, R. (1986). The diagnosis of schizophrenia by language analysis. British Journal of Psychiatry, 148, 275-278.

Frith, C.D. (1997). Language and communication in schizophrenia. En $\mathrm{J}$. France and N, Muir (Eds), Communication and the Mentally ill patient,(pp 10-17). London: Jessica Kingsley.

Frith, C.D. y Corcoran, R. (1996). Exploring theory of mind in people with schizophrenia, Psychological Medicine, 26, 521-530.

Frith, C.D., Leary, J., Cahill, C. y Johnstone, E.C. (1991). Performance en psychological tests. British Journal of Psychiatry, 159 (suppl,13) 26-29.

Garety, P.A. y Freeman, D. (1999). Cognitive approaches to delusions: A critical review of theories and evidence. British Journal of Clinical Psychology, 38, 113- 154.

González, E., Alonso del Teso, F., Mínguez, L y Sanguino, R. (2003). Deterioro ejecutivo y sintomatología en la esquizofrenia. Anales de Psiquiatría, 19 (10), 417-421.

Herold, R, Tényi, T, Lénárd, K y Trixler, M. (2002). Theory of mind deficit in people with schizophrenia during remission. Psychological Medicine, 32, 1125-1129.

Kay, S.R., Fiszbein, A. y Opler, L.A. (1987). The positive and negative syndrome scale (PANSS) for schizophrenia. Schizophrenia Bulletin, 13, 261-276.

King, K, Fraser, W.I., Thomas, P y Kennedy, R.E. (1990). Reexamination of the language of psychotic subjects. British Journal of Psychiatry, 156, 211-215.

Kruskal, J.B. y Wish, M. (1984). Multidimensional scaling. Beverly Hills, CA: Sage Publications.

Langdon, R y Coltheart, M. (2000). The cognitive neuropsychology of delusions. In M. Coltheart y M. Davies Pathologies of belief (pp 183216). Oxford. Blackwell Publishers. 
Langdon, R; Coltheart, M; Ward, P; Catts, S. (2002). Disturbed communication in schizophrenia: the role of poor pragmatics and poor mind-reading. Psychological Medicine, 32, 1273-1284.

Langdon, R, Michie, P.T., Ward, P.B., McConaghy, N, Catts, S.V., Coltheart, M.(1997). Defective self and / or other mentalising in schizophrenia: a cognitive neuropsychological approach. Cognitive Neuropsychiatry 2 (3), 167- 193.

Mazza, M; De Risio, A; Roncone, R; Casacchia,M. (2001). Selective impairments of theory of mind in people with schizophrenia. Schizophrenia Research, vol. 47 (2-3), 299-308.

Mendoza, E. (2001). Del TEL al autismo: El trastorno semánticopragmático (TSP). En E. Mendoza (Coord.), Trastorno específico del lenguaje (TEL) (pp 225-252). Madrid: Pirámide.

Mitchley, N.J.; Barber, J; Gray, J; Brooks, D.N.; Livingstone, M.G. (1998).

Comprehension irony in schizophrenia. Cognitive Neuropsychiatry, 3 (2), 127-138.

Moore, C., Pure, K. y Furrow, D. (1990). Children's understanding of the modal expression of speaker certainty and its relation to the development of a representational theory of mind. Child Development, 61 (3), 722-730.

Murray, R, O'Callaghan, E, Castle, D. y Lewis, Sh. (1992). A neurodevelopmental approach to the classification of schizophrenia. Schizophrenia Bulletin, 18 (2), 319-332.

Peralta, V. y Cuesta, M.J. (1994). Validación de la escala de los síndromes positivo y negativo (PANNS) en una muestra de esquizofrénicos españoles. Actas Luso Españolas de Neurología Psiquiátrica, 22 (4), 171-177.

Pickup, G. y Frith, C. (2001). Theory of mind impairments in schizophrenia: Symptomatology, severity and specifity. Psychological Medicine, 31 (2), 207-220.

Portela, M., Vírseda, A. y Gayubo, L. (2003). Revisión sobre el estudio de la teoría de la mente en trastornos generalizados del desarrollo y esquizofrenia. Actas Españolas de Psiquiatría, 31 (6), 339-346.

Real, J.E. (2001). Escalarniento multidimensional. Salamanca: Hespérides. Rips, L.J. y Conrad, F.G. (1989). Folk psychology of mental activities.

Psychological Review, 96, 187-207.

Sarfati, $Y(2000)$. Deficit of the theory of mind in schizophrenia: clinical 
concept and review of experimental arguments. Canadian Journal of Psychiatry, 45 (4), 363-368.

Sarfati, Y. y Hardy- Baylé, M.C. (1999). How do people with schizophrenia explain the behaviour of others? A study of theory of mind and its relationship to thought and speech disorganization in schizophrenia. Psychological Medicine, 29, 613-620.

Sarfati, Y., Hardy- Baylè, M.C., Besche, C. y Widlöcher, D. (1997a). Attribution of intentions to others in people with schizophrenia: A non- verbal exploration with comic- strip. Schizophrenia Research, 25, 199-209.

Sarfati, Y., Hardy- Baylé, M.C., Brunet, E. y Widlöcher, D. (1999). Investigating theory of mind in schizophrenia: Influence of verbalization in disorganized and non-disorganized patients. Schizophrenia Research, 37, 183-190.

Sarfati, Y., Hardy-Baylé, M.C., Nadel, J., Chevalier, J.F y Widlöcher, D. (1997b). Attribution of mental states to others in schizophrenic patients. Cognitive Neuropsychiatry, 2, 1-17.

Sarfati, Y, Passerieux, Ch , Hardy-Bayle, M.C. (2000). Can verbalization remedy the theory of mind deficit in schizophrenia?. Psychopathology, 33 (5), 246-251.

Schwanenflugel, P.J., Fabricius, W.F. y Alexander, J.M. (1994a). Developing theories of mind: Understanding concepts and relations among mental activities. Child Development, 65, 1546-1563.

Schwanenflugel, P.J., Fabricius, W.V. y Noyes, C.R. (1996). Developing organization of mental verbs: Evidence for the development of a constructivist theory of mind in middle childhood. Cognitive Development, 11, 265-294.

Schwanenflugel, P.J., Fabricius, W.V., Noyes,C.R., Bigler K.D. y Alexander, J.M. (1994b). The organization of mental verbs and folk theories of knowing. Journal of Memory and Language, 33, 376395.

Schwanenflugel, P.J., Henderson, R.L. y Fabricius, W.V. (1998) Developing organization of mental verbs and theory of mind in middle childhood: Evidence from extensions. Developmental Psychology, 34, 512-524.

Schwanenflugel, P.J., Martin, M. y Takahashi, T. (1999). The organization of verbs of knowing: Evidence for cultural commonality 
and variation in theory of mind. Memory and Cognition, 27 (5), 813825.

Shoben, E. J. y Ross, B.H. (1987). Structure and process in cognitive psychology using multidimensional scaling and related techniques. En R. Ronning, J. Glover, J. Conoley y J. Witt, The influence of cognitive psychology on testing (pp 229-266). New Jersey: Lawrence Erlbaum Ass.

Thomas, P, King, K, Fraser, W.I. y Kendell, R.E. (1990). Linguistic performance in schizophrenia: A comparison of acute and chronic patients. British Journal of Psychiatry, 156, 204-210. 